\title{
Analysis of the distribution of parameter of membrane lipid phase state
}

\author{
Nurminsky V.N. ${ }^{1 *}$, Bukin Yu.S. ${ }^{2}$, Ozolina N.V. ${ }^{1}$ \\ ${ }^{1}$ Siberian Institute of Plant Physiology and Biochemistry, SB RAS, Irkutsk, Russia \\ ${ }^{2}$ Limnological Institute, SB RAS, Irkutsk, Russia \\ *email: cell@sifibr.irk.ru
}

On the basis of the $\mathrm{R}$ programming language, a technique has been developed for analyzing data on the packing density (phase state, lipid order) of the membrane lipid phase obtained by measuring the generalized polarization (GP) of the fluorescence of probe laurdan. It is known that the fluorescence spectrum of laurdan changes depending on the ordering (microviscosity) of the lipid phase. The histograms of distributions of GP values reflect the degree of ordering of the lipid phase, and make it possible to reveal the presence of membrane heterogeneity (membrane rafts). The developed analysis allows constructing a theoretical multimodal distribution as a superposition (overlay) of several normal distributions using the method of least squares. The limitation on the number of components (modes) for describing the distribution of the GP value is conducted using two indicators of informative significance - the Akaike information criterion (AIC) and the Bayesian information criterion (BIC). The multimodal distribution with such a number of normally distributed modes (components), for which the value of the covariance coefficient $\mathrm{R}^{2}>0.99$, with the minimum values of AIC and BIC, was chosen as the optimal one. To test the technique, we analyzed the ordering of lipids in the raft membranes of mitochondrial membranes in halophytes Salicornia perennans Willd., Halocnemum strobilaceum Bieb. and Artemisia santonica L., differing in the salt tolerance. According to the analysis of lipid ordering, the membrane material of the mitochondria of $S$. perennans, H. strobilaceum, and A. santonica may contain raft structures. So, the analysis of data on the density (ordering) of the lipid phase of the membrane by measuring the GP fluorescence of the laurdan probe can be used to identify more densely packed raft structures in the membrane.

Acknowledgments: This work was carried out with partial use of the Russian Foundation for Basic Research, grant No. 19-04-00013. 